\section{artelogie}

\section{Artelogie}

Recherche sur les arts, le patrimoine et la littérature de l'Amérique latine

10 | 2017

Après le paysage : l'art, l'inscription et la représentation de la nature en Amérique latine aujourd'hui

\title{
La forme entre la culture et la nature : Phasmides de Daniel Steegmann Mangrané
}

Fábio Zuker

\section{(2) OpenEdition \\ Journals}

Édition électronique

URL : http://journals.openedition.org/artelogie/864

DOI : 10.4000/artelogie.864

ISSN : 2115-6395

Éditeur

Association ESCAL

Référence électronique

Fábio Zuker, «La forme entre la culture et la nature : Phasmides de Daniel Steegmann Mangrané ». Artelogie [En ligne], 10 | 2017, mis en ligne le 05 avril 2017, consulté le 21 avril 2019. URL : http:// journals.openedition.org/artelogie/864; DOI : 10.4000/artelogie.864

Ce document a été généré automatiquement le 21 avril 2019

Association ESCAL 


\title{
La forme entre la culture et la nature : Phasmides de Daniel Steegmann Mangrané
}

\author{
Fábio Zuker
}

«Quand je voyais un objet extérieur, la conscience que je le voyais restait entre moi et lui, le bordait d'un mince liséré spirituel qui m'empêchait de jamais toucher directement sa matière ;

elle se volatilisait en quelque sort avant que je prisse contact avec elle, comme un corps incandescent qu'on approche d'un objet mouillé ne touche pas son humidité parce qu'il se fait toujours précéder d'une zone d'évaporation »

Marcel Proust

\section{Questionner à travers les constructions formelles}

1 Daniel Steegmann Mangrané est né à Barcelone, en 1977. Il vit et travaille au Brésil depuis 2004. L'artiste a exposé dans plusieurs grands musées et galeries du monde entier, dont la $30^{\mathrm{e}}$ Biennale de São Paulo, le Museo de Arte Experimental El Eco (Mexique) et la FIAC à Paris (avec sa galerie Mendes Wood).

2 Le faire avec de l'anthropologie est bien présent dans notre texte, qui se construit à partir des œuvres de Steegmann Mangrané. C'est à partir des pièces comme Phasmides et $16 \mathrm{~mm}$ que nous démontrerons comment son œuvre arrive à questionner certaines séparations dichotomiques entre le monde de la culture et le monde de la nature. Ces tensions émergent dans la révisitation que ses travaux font de certains points promus par 
le mouvement anthropophagiste d'Oswald de Andrade et le néoconcrétisme de Lygia Clark, surtout son œuvre Bichos- mouvements qui ont largement marqué l'art au Brésil.

Daniel Steegmann Mangrané, Equal. 2008

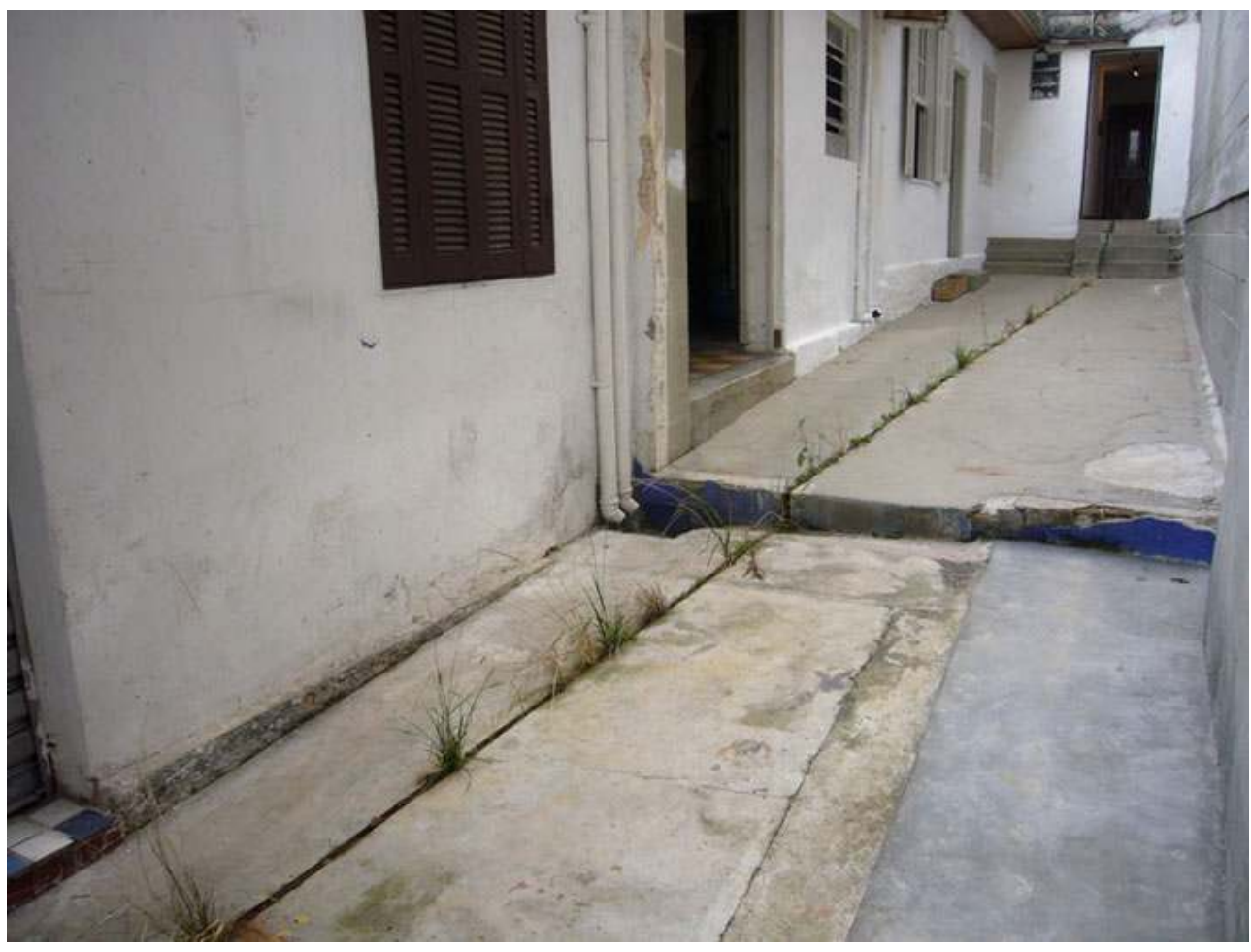

Coupure dans le sol, plants et Temps, 22 mètres dans l'Ateliê 397

3 Ces questionnements sur la séparation moderne entre nature et culture apparaissent dans plusieurs travaux de Steegmann Mangrané, toujours de manière formelle. Cela se traduit par la construction d'une opposition entre une forme naturelle chaotique d'un côté, et une forme culturelle, organisée, de l'autre. Dans l'intervention Equal, réalisée dans l'Ateliê 397 (centre d'art à São Paulo), cette opposition prend la forme d'une très fine coupure faite dans le ciment du sol, et dans laquelle des herbes ont poussé. Dans le film $16 \mathrm{~mm}$, c'est le chaos de la forêt qui est traversé par une caméra se déplaçant sur un cordeau d'acier, avec comme moteur sa propre bobine.

4 Cette opposition formelle entre les éléments culturels et naturels est l'objet d'une constante déconstruction. L'artiste semble toujours vouloir faire en sorte que la forme géométrique émerge comme organique, et que la forme organique se fasse visible comme géométrique, mais alors beaucoup plus complexe que l'autre. En bref, les formes ne sont pas définies par elles-mêmes, mais de façon relationnelle: Steegmann Mangrané essaie précisément de bouleverser ces distinctions simplistes entre les formes géométriques comme produit du travail humain d'une part et les formes organiques naturelles d'autre part. 


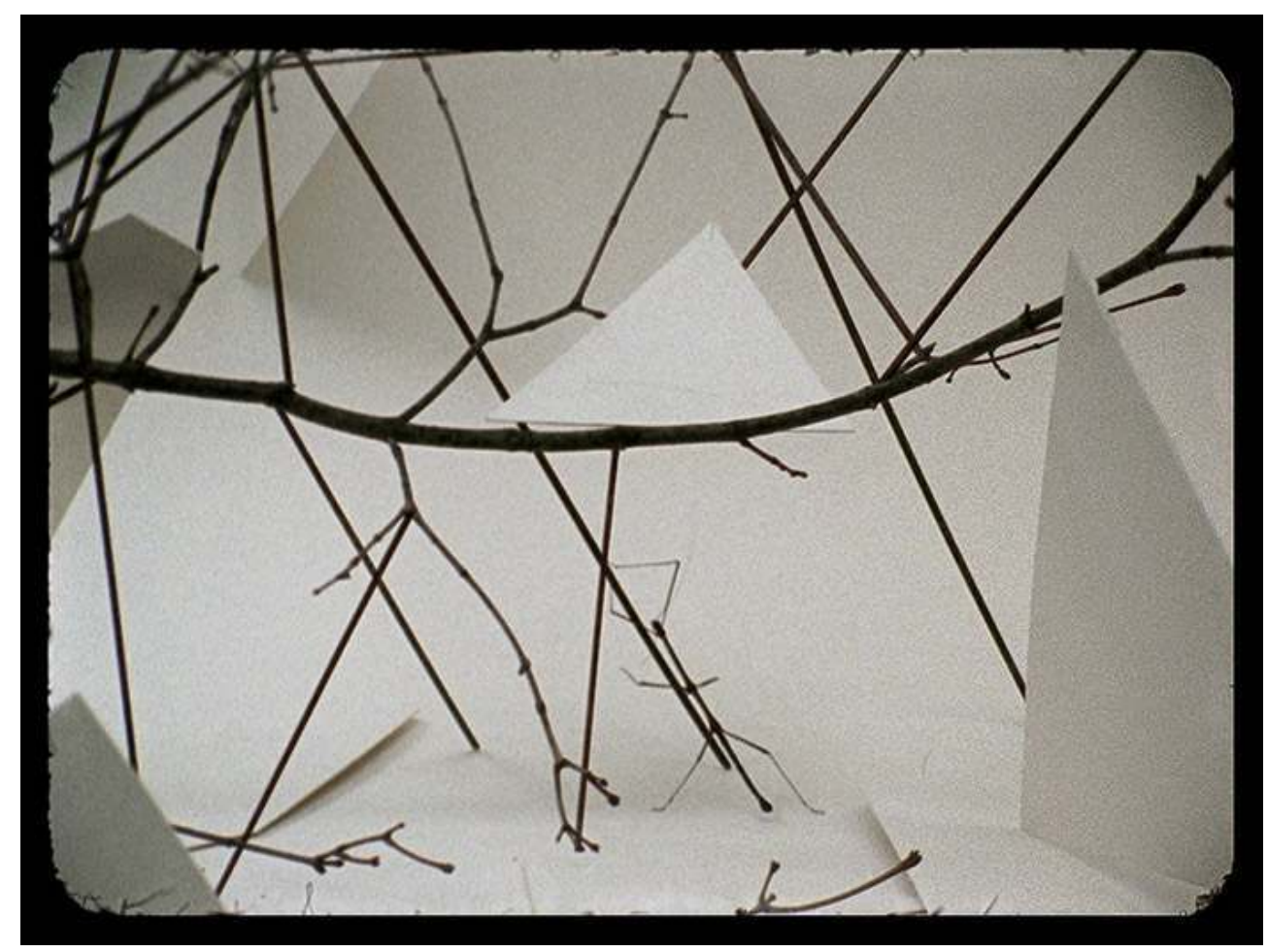

16 mm, "Batôn du diable", figures geométricisées et bois, 22'41"

5 La série Phasmides construit précisément ce rapport entre les formes à partir du jeu de camouflage d'un insecte de la famille des phasmes, aussi connu par son nom vernaculaire de «Bâton du Diable ». La série de Steegmann Mangrané consiste en un assemblage de vidéo, peintures, hologrammes imprimés en 3D et une sculpture à grande échelle qui ressemble à la physionomie de l'animal.

6 Dans les vidéos et dans les photographies, le phasme tantôt se cache dans la nature, tantôt dans des constructions humaines. Ainsi, il est presque impossible de voir la singularité de sa constitution physique: les structures physiologiques de l'animal se construisent dans ses œuvres à partir des matières contre lesquelles l'animal est positionné. Les peintures, à leur tour, deviennent des abstractions formelles à partir du contraste avec l'animal et donnent naissance à des formes géométriques, qui aboutissent à la sculpture géométrisée au centre de la salle d'exposition.

7 Cette série a été suscitée par une expérience personnelle de l'artiste avec cet insecte dans le Museu do Açude, à Rio de Janeiro. Après l'avoir aperçu sur la surface d'une piscine vidée, l'animal a disparu parmi d'autres bâtons. Une expérience très subtile qui, selon Steegmann Mangrané, a marqué sa façon de penser les images.

8 Phasmides est le nom d'une famille d'insectes capables de se mélanger avec des bâtons et des racines. Le terme a la même racine étymologique que le mot fantôme, qui signifie, en réalité, «apparition». Ce jeu d'apparition et disparition s'organise dans l'installation autour d'un animal parfois camouflé entre des « objets naturels » (même si enlevés à leurs environnements), ou entre des "objets artificiels» (formes géométriques abstraites), construites par l'artiste. Ainsi, le « Bâton du Diable » s'insère-t-il dans un questionnement sur le statut de l'image: comment une image peut être si forte et si faible en même 
temps ? Comment peut-elle exister dans une zone limitrophe entre le monde animal et le monde végétal? Il s'agit d'un animal, sans aucun doute, qui cherche à ressembler à un objet naturel. Dans cette série, les réflexions de l'artiste se construisent aussi autour du moyen par lequel la pellicule du film est faite, la cellulose. Il en résulte une sorte de fragilité inhérente à l'image, due à sa fixation sur un élément physique fait du même matériau que celui que le phasme mime : la matière organique composée par la cellulose.

9 Steegmann Mangrané raconte l'influence que la lecture d'un texte de George DidiHuberman, intitule The Paradox of the Phasmide, a eu sur ses perceptions des modes de fonctionnement de l'image. L'auteur raconte une histoire assez proche de celle de Steegmann Mangrané, qui a eu lieu au Jardin des Plantes, à Paris, et où l'animal a disparu et réapparu en fonction du fond devant lequel il était.

Daniel Steegmann Mangrané, Phasmides, 2008-2012

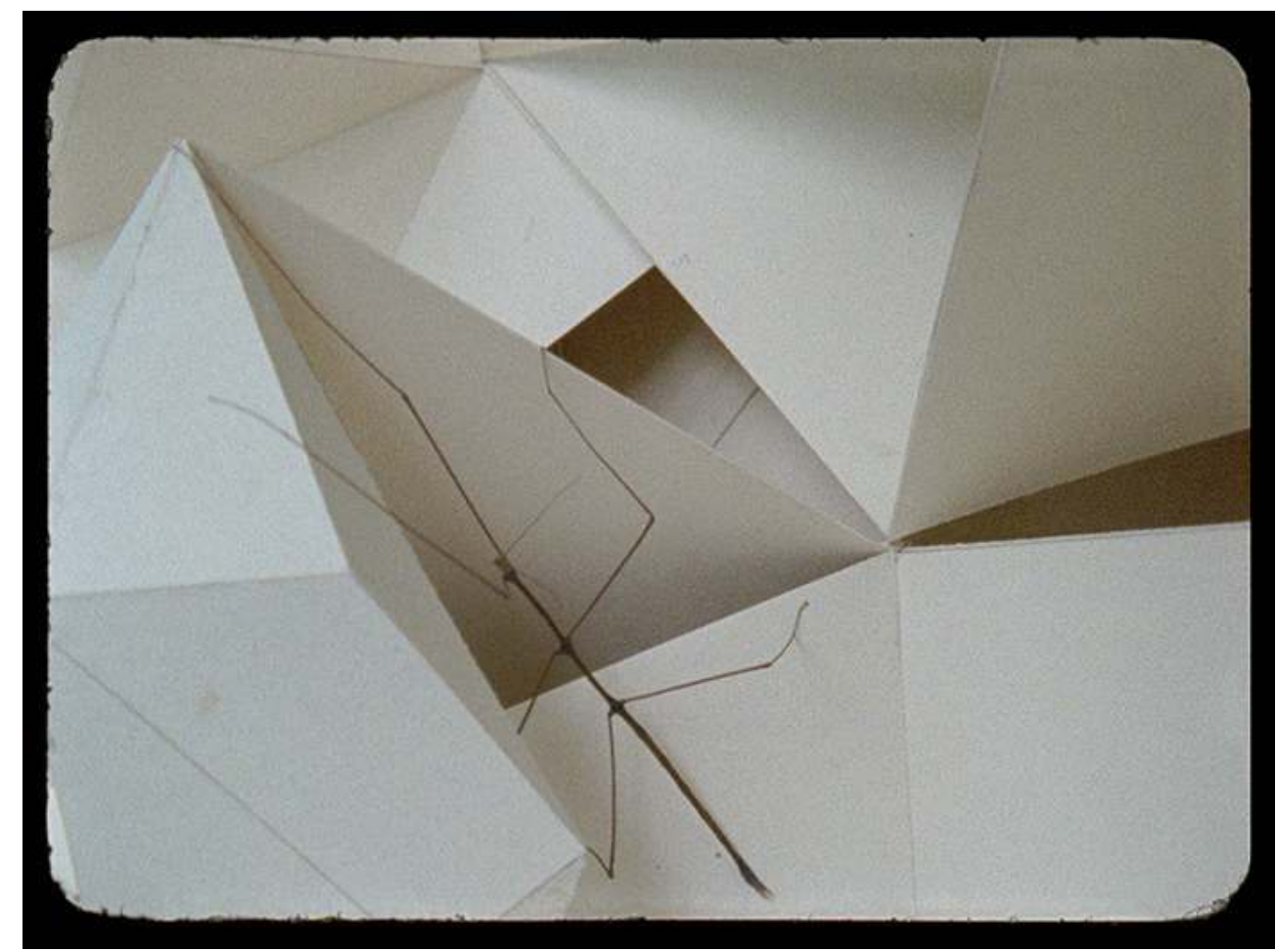

16 mm, "Batôn du diable", figures géométrisées et bois, 22'41"

10 Ce rapport entre la figure et le fond gagne, à travers l'œuvre de l'artiste, un statut de « mode de fonctionnement de l'art » : la figure doit être placée contre un fond spécifique pour devenir significative. Au-delà du cas spécifique du phasme, nous pouvons voir dans ce travail une métaphore de la façon dont l'art opère. Il n'existe en effet pas tout seul, mais en rapport avec ce qui l'entoure, dans un étrange et paradoxal jeu entre certaines choses qui doivent disparaître pour que d'autres puissent apparaître :

"The only things that appear are those which are first able to dissimulate themselves. Things already grasped in their aspect or peacefully resembling themselves never appear. They are apparent, of course, but only apparent: they will never be given to us as appearing. What then is necessary for an apparition, the event of appearing? What must happen just before appearing closes itself within a presumably stable or hopefully definitive aspect? There must be a unique and momentary opening that will mark the 
apparition as an apparition. A paradox bursts forth because, in the very moment that it opens itself to the visible world, appearing is destined to be something like dissimulation. A paradox bursts forth because, for but a moment, appearing gives access to the here below, to something that suggests the contrary or, better yet, the hell of the visible world - the realm of dissemblance » (DIDI-HUBERMAN, 1989).

Le phasme prend la relève dans des scénarios naturels et géométriques: il se cache (quand il s'arrête) et il réapparait (quand il bouge) dans les deux. Ce sont les rapports entre figure et fond, si importants dans le cinéma, dans la peinture ou dans la photographie, qui sont ici utilisés pour penser des questions plus vastes sur l'art en général, le langage et la forme: un signe doit être transparent pour transmettre un signifié - il n'est lisible que lorsqu'il est transparent. Quand il devient opaque, nous restons attachés à sa réalité physique, sans pouvoir voir au travers du signe. Ainsi, le phasme devient lisible ou opaque en fonction de l'objet dont il est rapproché, ou de ses propres mouvements. Tout se passe comme si les choses n'avaient pas de stabilité intrinsèque.

Daniel Steegmann Mangrané, Holograma 2 (estrutura e galho). Série Phasmides, 2015

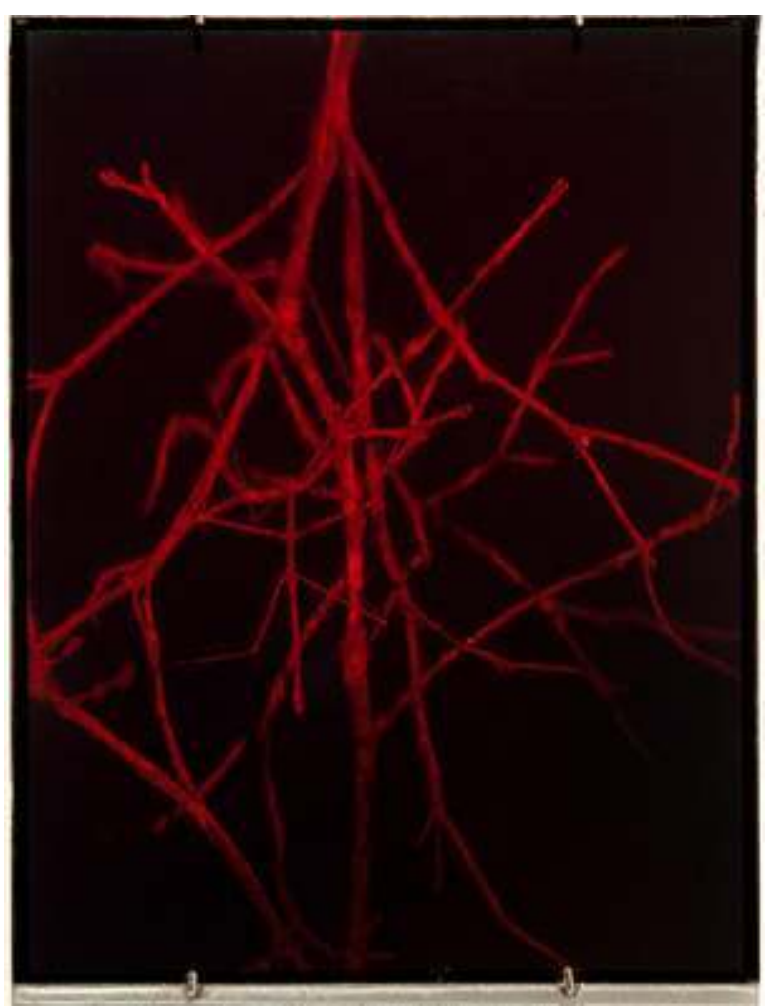

Hologramme, $25 \times 19 \mathrm{~cm}$

Dans le livre Métaphysiques Cannibales, l'anthropologue brésilien Eduardo Viveiros de Castro met en évidence le fonctionnement interne de la discipline anthropologique. Pour lui, la création de connaissance dans l'anthropologie est bien plus liée à la cosmologie des peuples étudiés, qui ne seront plus des objets à être étudiés, mais qui ont précisément un pouvoir d'agencement. En opposition à la thèse postmoderne selon laquelle les non-occidentaux étaient les créations d'un jeu de forces de l'Occident, sans voix dans les processus de transformation et formulation de l'anthropologie, 
Viveiros de Castro affirme que «toutes les théories anthropologiques non triviales sont des versions des pratiques de connaissance indigène » (VIVEIROS DE CASTRO, 2009 : p. 6).

14 Comprendre l'altérité de l'autre, pour critiquer et nous faire repenser nos propres pratiques. Voici un procédé cher à cette branche de l'anthropologie contemporaine (qui d'une certaine façon structure toute notre recherche), et qui retrouve dans ces travaux de Steegmann Mangrané une résonance unique. Dans cette série, c'est la procédure d'apparition et de camouflage du phasme dans son environnement qui devient le mode d'opération de l'œuvre, et qui fonctionne de façon analogue à l'anthropologie contemporaine : l'œuvre ne représente pas le phasme, mais mime plutôt une procédure qui est structurante de son mode d'existence. Il ne s'agit pas, bien entendu, d'affirmer que l'artiste fait l'illustration d'une certaine procédure anthropologique, mais de comparer les résonances entre ces deux procédures trouvant leurs origines dans des champs si différents.

15 Ce qui caractérise l'anthropologie, selon l'écossais Tim Ingold, c'est exactement le dialogue et l'étude faits avec l'autre, qui nous apprennent d'autres modes de connaissance $\mathrm{du}$ monde, ou selon un point de vue plus radical, nous ouvre des portes sur d'autres mondes :

"What truly distinguishes anthropology, I believe, is that it is not a study of at all, but a study with. Anthropologists work and studies with people. Immersed with them in an environment of joint activity, they learn to see things (or hear them, or touch them) in the way their teachers and companions do. An education in anthropology, therefore, does more than furnish us with knowledge about the world - about people and their societies. It rather educates our perception of the world, and opens our eyes and minds to other possibilities of being. The questions we address are philosophical ones [...] But it is the fact that we address these questions in the world, and not from the armchair - that this world is not just what we think about, but what we think with, and that in its thinking the mind wanders along pathways extending far beyond the envelope of the skin - that makes the enterprise anthropological and, by the same token, radically different from positivist science. We do our philosophy out of doors. And in this, the world and its inhabitants, human and non-human, are our teachers, mentors and interlocutors " (INGOLD, 2008: 82-83) 
Daniel Steegman Mangrané, Holograma 6 (estrutura e fora. Série Phasmides, 2013

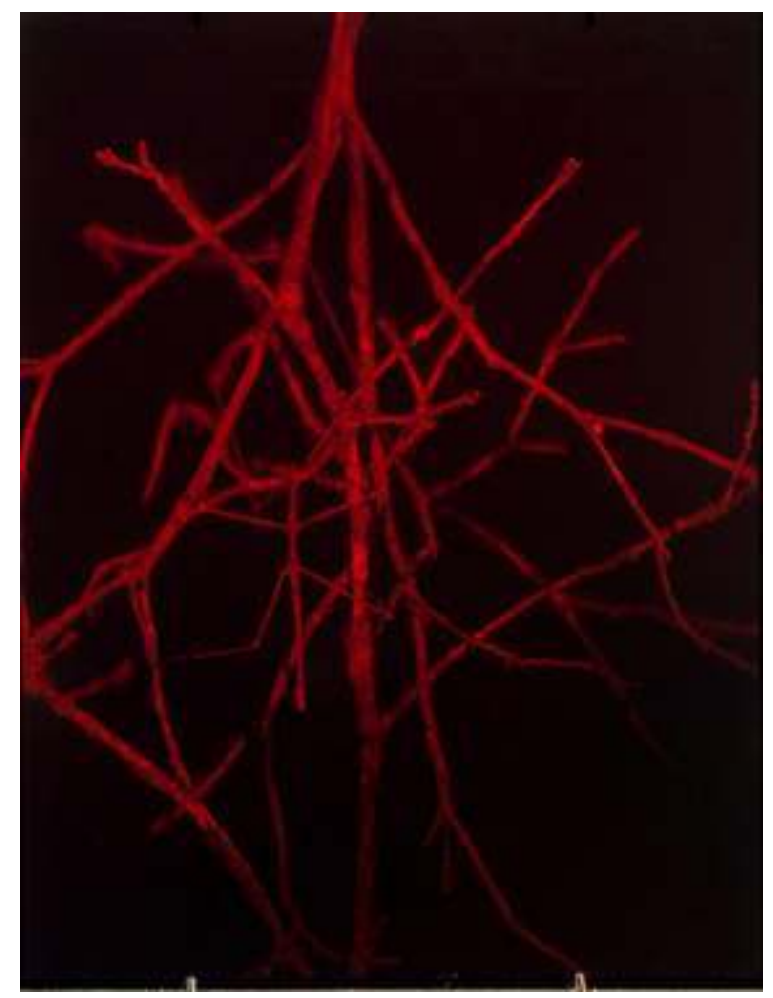

Hologramme, $25 \times 19 \mathrm{~cm}$

16 Les idées amérindiennes avec lesquels travail Eduardo Viveiros de Castro ont un statut ontologique tout autre, qui met en perspective nos propres divisions entre nature et culture. De la sorte, elles mettent en perspective la propre notion d'une culture qui peut varier autour d'une nature donnée. Dans la proposition du perspectivisme (qui se construit comme une version d'une théorie native amérindienne), il n'existe pas des sujets ni des objets en soit. Si, dans l'Occident (de façon assez générale et abstraite), nous nous comprenons à partir d'un système d'oppositions dans lequel le monde est divisé entre nature et culture, des animaux et des animaux spéciaux (les humains), chez les amérindiens l'opposition elle-même n'existe pas.

17 Prenons comme exemple une situation assez courante dans l'Occident moderne. Imaginons un Français, un Anglais, un Espagnol, un Italien et un Portugais tous réunis autour d'un verre, chacun pouvant nommer dans sa langue l'objet qu'ils ont devant eux et qui s'appelle alors « glass ", "vaso », «bichieri » ou " copo » : il ne fait aucun doute que tous sont devant un objet qui a une existence indépendante de celui qui le regarde. En revanche, pour les amérindiens, c'est l'humanité même qui est partagée et non pas l'animalité - leur monde étant beaucoup plus peuplé que chez les occidentaux. C'est pourquoi nous pouvons parler d'un rapport autre entre sujet et objet. Il devient impossible de dire qu'un objet existe en tant que tel, parce que les rapports se constituent toujours à partir d'une négociation entre les sujets.

18 Ainsi, si nous n'avons pas des sujets d'un côté et des objets de l'autre, il ne reste guère de rapport entre les spectateurs et les œuvres d'art: tout devient un processus de transformation incessante, des combinaisons d'agents qui s'influencent mutuellement. Ce qui fait que les rapports entre les choses, entre choses et sujets et entre sujets et sujets 
deviennent plus compliqués, et mettent en perspective nos propres catégories occidentales modernes d'appréhension de la réalité.

\section{Face au néoconcretisme}

Dans la lecture que nous faisons de la série Phasmides, la manière dont la tradition néoconcrétiste brésilienne est relue joue un rôle central. Nous y voyons une évidente proximité avec l'œuvre Bichos, de Lygia Clark. Selon le critique Ronaldo Brito, la série de Lygia Clark se constitue à partir d'une tentative de diluer l'objet, de proposer des mutations organiques, une « rupture avec la forme du monde, de l'idée occidentale deux fois millénaire de l'être en tant que figure stable » (BRITO, 1994, p. 28).

D'autre part, selon le critique, la série de l'artiste est aussi une expérience dissolvante, opposée à celle de la construction. Ce sont des sculptures transitives, relationnelles. C'est la stabilité de la figure qui est ici mise en évidence, à partir des plaques de métal attachées les unes aux autres et qui forment des objets ressemblant à certains animaux elles sont manipulées par le spectateur, qui leur donne des formes diverses.

Steegmann Mangrané porte cette procédure à un autre niveau. Tout l'être est compris comme un processus: nos conceptions de ce qu'est une sculpture sont radicalement distinctes des conceptions à l'époque de Lygia Clark - et ce en certaine partie grâce à elle. Cette instabilité de l'objet, cette transitorialité de la forme dans son milieu, dans la série de Steegmann Mangrané, propose une réflexion sur nos propres visions de l'objet, et de l'opposition entre culture et nature - structurante de la pensée occidentale moderne.

Daniel Steegmann Mangrané, Escultura "forma modelo". Serie Phasmides, 2013

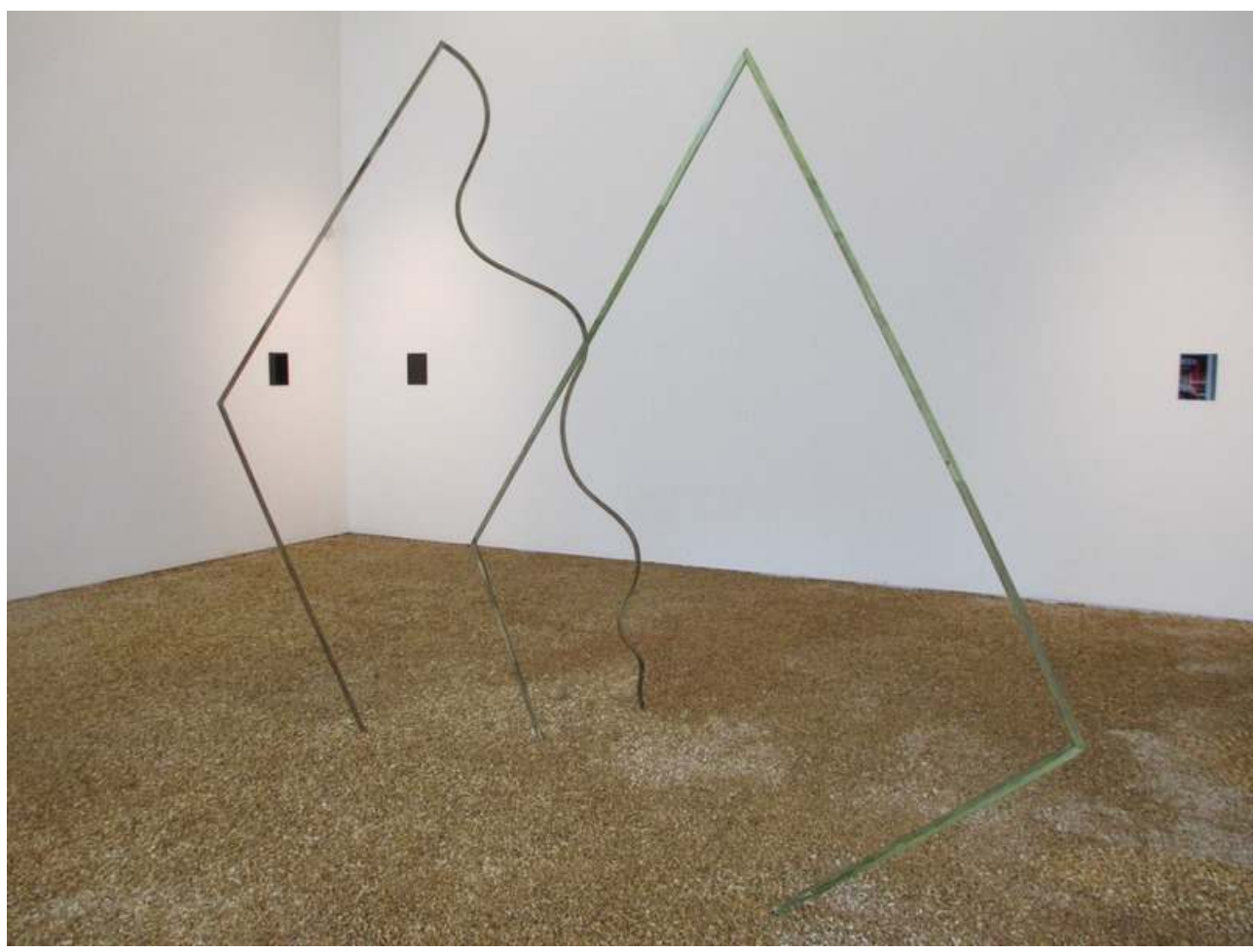

Sculpture, $272 \times 340 \times 150 \mathrm{~cm}$ 
La critique de la rationalité moderne par la mise en examen des séparations entre nature et culture est déjà présente dans les écrits de Roger Caillois. Dans le texte Mimétisme et Psychasthénie Légendaire (CALLOIS, 1935), l'intellectuel voit dans le phénomène du camouflage et de la disparition animale un désir de se fondre dans l'environnement qui l'entoure. Pas comme une technique de défense, mais plutôt comme un désir mythologique de disparaître dans le monde. Pour Steegmann Mangrané, ses travaux artistiques, mais aussi le langage, les danses, et les arts visuels en général, n'auraient-ils pas eux aussi ce désir implicite de dissolution du corps/objet dans le monde?

23 En conséquence, dans la série de l'artiste nous, en tant que spectateurs et humains, sommes placés dans cette zone limitrophe d'existence des phasmes, cette zone d'indéfinition de l'espace. Cela est suggéré par une sculpture qui se trouve au centre de l'installation, similaire aux structures présentes dans les vidéos, et qui nous invite à vivre une expérience similaire à celle des phasmes. L'expérience personnelle du spectateur retrouve, ainsi, sa proposition conceptuelle. Comme l'affirme l'artiste, dans l'entretien que nous avons réalisé avec lui au cours de notre recherche: "Je cherche toujours le moment où le spectateur ne regarde plus les travaux, mais sa propre expérience; le moment où il se regarde lui-même ».

Daniel Steegmann Mangrané, 16mm, 2008-2011

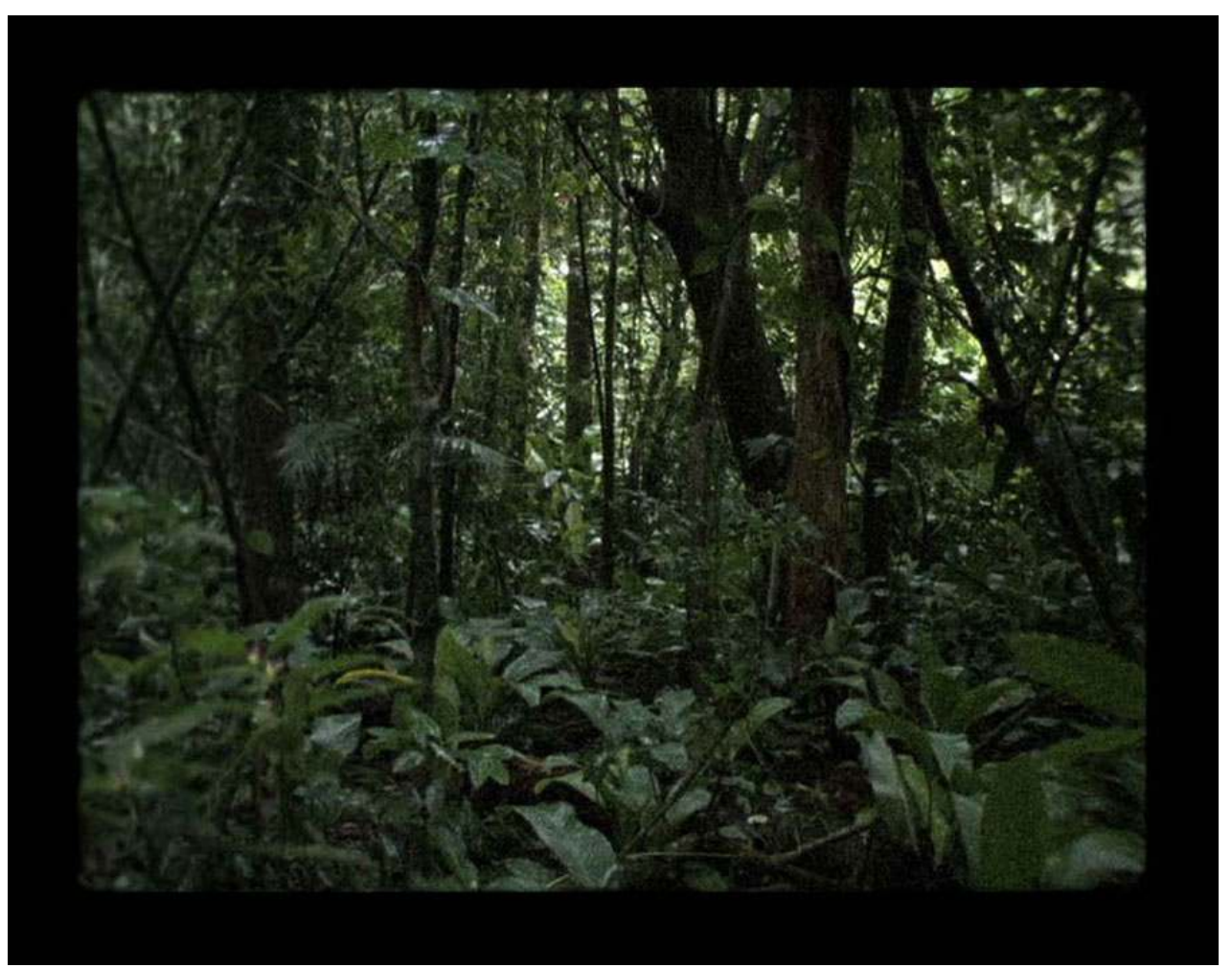

Prise du vidéo $16 \mathrm{~mm}$, couleur, 5'26"

24 Tout compte fait, il nous faut souligner que notre intérêt pour ces procédures formelles des œuvres de Steegmann Mangrané est capable de mettre en évidence cette déjà nommée séparation entre nature et culture. Cette manière de penser par des formes est aussi constitutive des films faits par l'artiste. Le film présent dans la série Phasmides est ainsi constitué entièrement en cellulose: le scénario est fait en bois, les formes 
géométriques en papier, le négatif du film est lui-même en cellulose, et l'animal (le phasme) veut devenir du bois, cellulose.

25 Nous devons lire ce travail en rapport avec le film $16 \mathrm{~mm}$ : sur une ligne étroite d'acier tendue à l'intérieur d'une forêt dense, une caméra bouge sur la corde et s'insère dans la nature à partir du mouvement de sa bobine interne. Et voilà que nous sommes de nouveau face à la confrontation initiale traitée dans notre texte, celle de la déconstruction de la dichotomie entre une forme naturelle et chaotique d'un côté, et une forme culturelle et organisée de l'autre.

Daniel Steegmann Mangrané, 16mm, 2008-2011

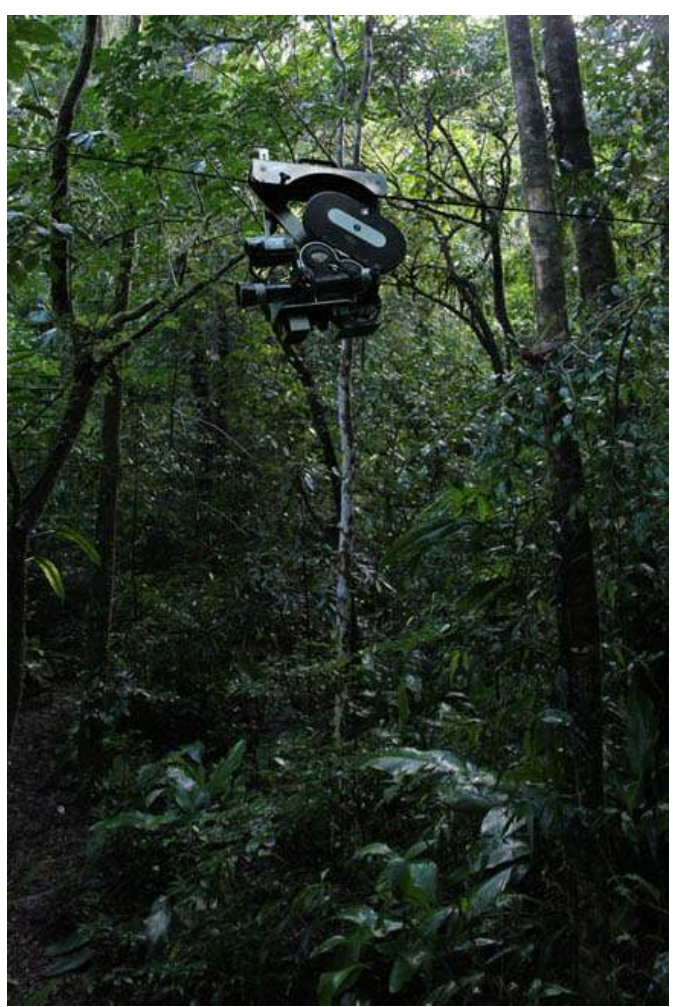

Vidéo 16 mm, couleur, 5'26"

26 Le cinéma et l'anthropologie sont tous les deux influencés par les mêmes pouvoirs coloniaux et aussi certaines motivations en commun. Entre la fin du XIX ${ }^{e}$ siècle et début du $\mathrm{xx}^{\mathrm{e}}$ ils partagent ainsi une structure épistémologique : tous les deux veulent créer une certaine narrative réelle, véritable (PIAULT, 2000). À cette fin, il faut que l'objet étudié devienne un document neutre. Ainsi, dans les premiers films anthropologiques, il s'agissait de cacher le moment où le réalisateur dirigeait les personnages et montait sur scène. Simplifier et nettoyer l'image, enlever tout ce que peut perturber le processus de création et de présentation de la réalité.

27 Tout cela est visible dans la vidéo présente dans la série Phasmides : la caméra finit par dénoncer l'artificialité de l'expérience et du scénario, lorsqu'elle sort du cadre pour enregistrer l'atelier, lieu du tournage.

28 Ses œuvres tentent de critiquer les logiques d'un discours occidental qui, comme dans les films ethnographiques, essaient d'effacer le fait que ce qui est perçu comme une réalité par le spectateur est en réalité le résultat d'un processus contradictoire de négociations 
et tensions autour des conceptions sur le signifié de la culture et de la nature. Si une certaine conception du pouvoir a besoin d'une image nettoyée et cristallisé du réel pour exister, la recherche de Stegeemann Mangrané se construit exactement dans le point où les conceptions divergentes du réel sont en dispute.

\section{BIBLIOGRAPHIE}

BRITO Ronaldo, Experiência crítica, São Paulo, Editora Cosac Naify, 1994, 28. [Traduction faite par nous-même].

DIDI-HUBERMAN Georges, The Paradox of the Phasmid, 1989. http://www.usc.edu/dept/comp-lit/ tympanum/3/phasmid.html

INGOLD Tim, « Anthropology is not ethnography », in Proceedings of the British Academy, 154, The British Academy, 2008.

PIAUlt Marc-Henri, Anthropologie et Cinéma. Passage à l'image, passage par l'image, Paris, Nathan Cinéma, 2000.

VIVEIROS DE CASTRo Eduardo, Métaphysiques Cannibales, Paris, PUF, 2009.

\section{RÉSUMÉS}

Cet article essaie de faire émerger certaines réflexions à partir d'une lecture attentive, inspirée par la description anthropologique, des travaux de l'artiste Daniel Steegmann Mangrané, qui se concentrent sur les présupposés logiques de la distinction entre nature et culture. La vision de l'homme comme étant un animal, mais un animal spécifique, séparé des autres animaux et des objets, est à la base de l'imagination que la modernité a développée à propos d'elle-même. Ici, il s'agit d'étudier comment les œuvres de Steegmann Mangrané arrivent à expérimenter d'autres façons de comprendre le rapport entre nature et culture, en liaison avec la pensée des peuples amérindiens au Brésil. Cet article s'est aussi inspiré des entretiens que l'auteur a pu faire avec l'artiste.

Este artigo tenta fazer emergir algumas reflexões, a partir de uma leitura atenta e inspirado pela descrição antropológica, do trabalho do artista Daniel Steegmann Mangrané, centrando-se para isso nospressupostos lógicos da distinção entre natureza e cultura. A visão do homem como um animal, mas um animal específico, separado dos outros animais e objetos, encontra-se na base da imaginação que a modernidade tem desenvolvido sobre si mesma. Aqui, trata-se de estudar como as obras de Steegmann Mangrané experimentam outras formas de compreender a relação entre natureza e cultura, em conexão com o pensamento dos povos indígenas no Brasil. Este artigo é inspirado por entrevistas que o autor pode realizar com o artista. 
INDEX

Mots-clés : art contemporain, anthropologie, perspectivisme amérindien, décolonisation poststructuralisme

Palabras claves : arte contemporáneo, antropologia, perspectivismo ameríndio, descolonização, pós-estruturalismo

\section{AUTEUR}

\section{FÁBIO ZUKER}

Master en Sciences Humaines et Sociales - EHESS

Mention Théorie et Pratique du Langage et des Arts

Fabiozuker[at]gmail.com 